\title{
Force spectroscopy reveals the presence of structurally modified dimers in transthyretin amyloid annular oligomers
}

Abbreviations: AFM, atomic force microscopy; TTR, transthyretin; nTTR, native transthyretin; aoTTR, transthyretin amyloid annular oligomers; WT, wild type

\begin{abstract}
Toxicity in amyloidogenic protein misfolding disorders is thought to involve intermediate states of aggregation associated with the formation of amyloid fibrils. Despite their relevance, the heterogeneity and transience of these oligomers have placed great barriers in our understanding of their structural properties. Among amyloid intermediates annular oligomers or annular protofibrils have raised considerable interest because they a mechanism of cellular toxicity via membrane permeation. Here we investigated, by using AFM force spectroscopy, the structural detail of amyloid annular oligomers from transthyretin (TTR), a protein involved in systemic and neurodegenerative amyloidogenic disorders. Manipulation was performed in situ, in the absence of molecular handles and using persistence length-fit values to select relevant curves. Force curves reveal the presence of dimers in TTR annular oligomers that unfold via a series of structural intermediates. This is in contrast with the manipulation of native TTR that was more often manipulated over length scales compatible with a TTR monomer and without unfolding intermediates. Imaging and force spectroscopy data suggest that dimers are formed by the assembly of monomers in a head-to-head orientation with a nonnative interface along their $\beta$-strands. Furthermore, these dimers stack through nonnative contacts that may enhance the stability of the misfolded structure.
\end{abstract}

\section{KEYWORDS}

amyloid, annular oligomers, annular protofibrils, atomic force microscopy, force spectroscopy, transthyretin

National Research, Development and Innovation Office, VKSZ_14-1-2015-052. Hungarian Science Foundation, OTKA K109480.

\section{I INTRODUCTION}

Amyloid aggregates are among some of the most deleterious and frequent agents of protein folding diseases including disorders with neurological, ${ }^{1}$ cardiac, ${ }^{2,3}$ or systemic manifestations. ${ }^{3}$ In the typical end stage of amyloidogenesis micrometer-long amyloid fibrils deposit and accumulate in various tissues of the patients. ${ }^{3}$ This is the case with transthyretin (TTR), a homotetrameric serum-circulating protein involved in the transport of thyroxin and the cotransport of retinol. ${ }^{4}$ F1 The TTR monomers (Figure 1) contain a single $a$-helix and $2 \beta$-sheets, each composed of $4 \beta$-strands (DAGH and CBEF), and display a so called " $\beta$-sandwich" fold that resembles a "flattened $\beta$-barrel" with its $2 \beta$-sheets packed together mostly through hydrophobic interactions of its side chains. ${ }^{5}$ A TTR dimer is formed through hydrogen bonding across $\mathrm{H}-\mathrm{H}^{\prime}$ and $\mathrm{F}-\mathrm{F}^{\prime}$ chains between 2 TTR monomers, and the final tetrameric arrangement results from $\hat{2}$ dimers that face each other through the DAGH $\beta$-sheet in a 2 -fold symmetric fashion. ${ }^{5}$ The dimer-dimer interface in the equatorial plane of the tetramer delineates the contours of a pair of cavities for thyroxin binding. The binding of thyroxin and other similar small ligand molecules further stabilizes the tetrameric arrangement. ${ }^{6}$

To some extent the wild-type form of TTR appears to be prone to amyloid aggregation as evidenced by cases of senile systemic amyloidosis where WT TTR is found in amyloid deposits. ${ }^{7}$ However, certain mutations in the TTR sequence can greatly enhance the process of aggregation even though no particular amyloidogenic hotspot can be assigned. ${ }^{1,8}$ In spite of this, in vitro aggregation of WT TTR and of most of its amyloidogenic variants is often induced by mild acidification," which has been proposed to mimic the lysosomal environment associated with the conversion of proteins and peptides into their amyloidogenic form..$^{9-11}$ The resulting aggregates typically bind 


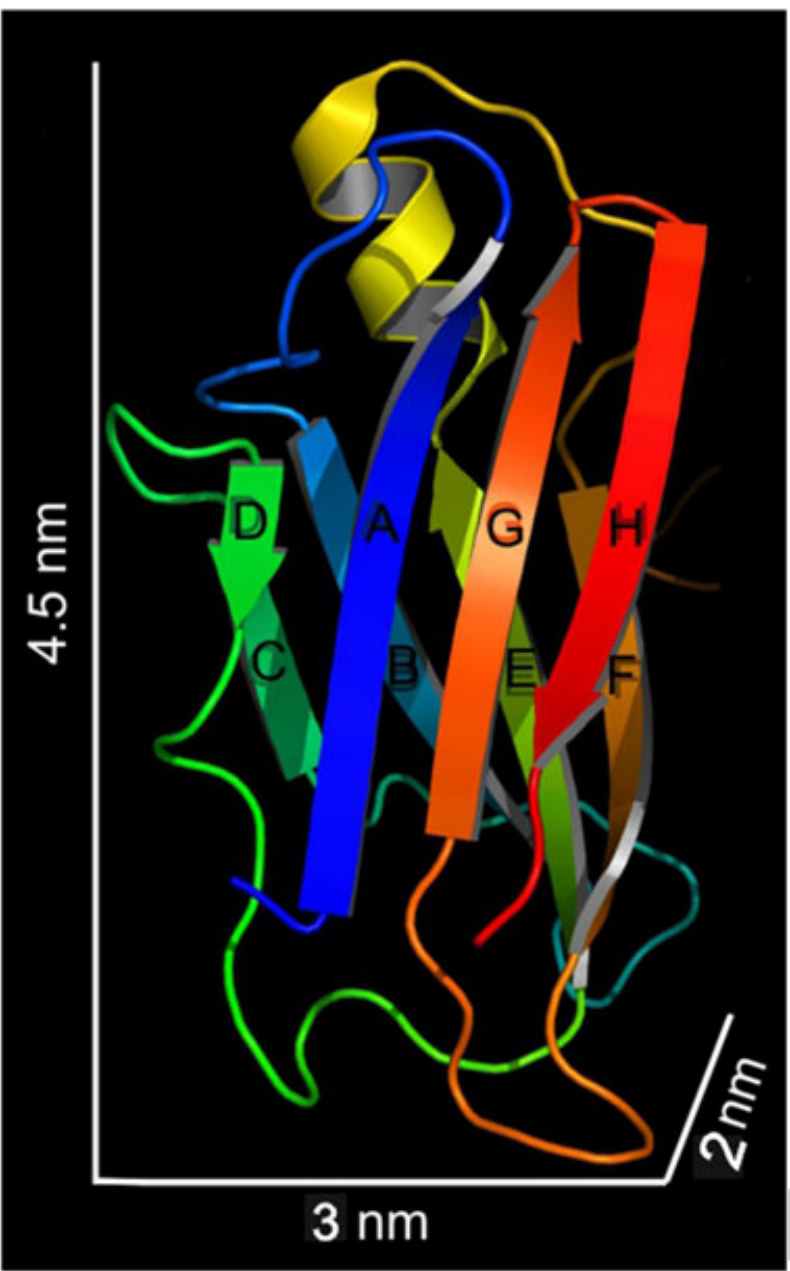

FIGURE 1 A 3D structure of transthyretin (TTR) monomer (PDB entry: $2 \mathrm{PAB}$ ) shows a beta-sandwich conformation by assembly of $8 \beta$-strands (A-F) into $2 \beta$-sheets: DAGH and CBEF

the amyloid markers Thioflavin-T and Congo red, ${ }^{12,13}$ display a high content of $\beta$-sheets, ${ }^{14}$ but lack the morphology of fibrils extracted from patient samples. ${ }^{12,15}$

While partial unfolding of the TTR monomer and disassembly of the tetramer is a prerequisite for fibrillation, ${ }^{16,17}$ there is strong debate over the details of the rest of the aggregation pathway. Some studies suggest that in vitro fibrillation is driven through the addition of monomers, ${ }^{12,16,18}$ while others have highlighted the role of oligomeric species as active participants in the fibrillogenesis process. ${ }^{13,14,19,20}$ Amyloid oligomers from different proteins have been proposed to be off-pathway oligomeric assemblies ${ }^{13,21}$ that share certain structural characteristics with amyloid fibrils, yet do not directly seed the fibrils. $^{21,22}$ Nonetheless, amyloid oligomers are viewed with extreme interest because they are likely to be a main source of amyloid toxicity in vivo. $2 \hat{3,24}$

While the mechanisms of amyloid toxicity remain unclear for the most part, interference with the cell membrane appears to be required. ${ }^{10,25}$ Amyloid fibrils have been increasingly recognized as a condensed state of misfolded proteins, which is less harmful than the amyloid oligomers associated with them. ${ }^{24,25}$ Among the different types of amyloid oligomers, ${ }^{25}$ those with annular morphology, termed either as annular oligomers or annular protofibrils, have received significant attention. They have been described across a variety of amyloid systems, and because toxicity appears to develop through poration of the cell membrane, annular oligomers have emerged as possible candidates behind amyloid toxicity. ${ }^{26,27}$ However, their transient nature ${ }^{19}$ together with the polymorphism associated with amyloid protein aggregation has placed great barriers toward understanding their molecular structure and properties.

Here we have used atomic force microscopy (AFM)-based force spectroscopy to obtain greater insight into the structure of TTR annular oligomers. We observed that they unfold via a series of intermediates, which we have characterized for forces associated with contourlength increments. Our data allow us to put forward a structural model for the organization of the TTR monomer within the annular assembly.

\section{2 | MATERIALS AND METHODS}

\section{1 | Sample preparation}

Recombinant WT TTR expressed in BL21 E coli, was isolated as described previously ${ }^{28}$ and further purified to high purity by using anion exchange (MonoQ column, GE Healthcare) and size exclusion (Superdex S75 column, GE Healthcare) chromatography steps. ${ }^{13}$ Stock solutions of WT TTR of 4 to $8 \mathrm{mg} / \mathrm{mL}$ were kept in $10 \mathrm{mM}$ HEPES, $\mathrm{pH} 7.0$ at $-20^{\circ} \mathrm{C}$. Protein quantification was performed by spectrophotometry at $280 \mathrm{~nm}\left(\varepsilon=77600 \mathrm{M}^{-1} \mathrm{~cm}^{-1}\right)$. The TTR amyloid aggregation was induced by diluting TTR to a final concentration of $1 \mathrm{mg} / \mathrm{mL}$ in $50 \mathrm{mM}$ sodium acetate buffer at $\mathrm{pH} 3.6$ and incubating at $37^{\circ} \mathrm{C} .^{13,19}$ Typically, within $24 \mathrm{~h}$ of incubation transient oligomeric assemblies with annular shape were observed as reported previously. ${ }^{19}$

\section{2 | Atomic force microscopy and force spectroscopy}

Data acquisition was performed in liquid and at room temperature with an MFP-3D Atomic Force Microscope (Asylum Research, Santa Barbara, USA), using a cantilever (Biolever A, Olympus) with a resonance frequency in liquid of $\sim 9.2 \mathrm{kHz}$. Noncontact (AC) mode AFM images were acquired using free and set-point amplitudes of $\sim 0.3$ and $\sim 0.2 \mathrm{~V}$, respectively. Images of $1024 \times 512$ and $512 \times 512$ pixels were obtained at a line-scanning frequency of $\sim 0.8 \mathrm{~Hz}$. Typically, 3 hours prior to probing the sample by AFM, $1 \times 1 \mathrm{~cm}^{2}$ of $\mathrm{V}-1$ grade muscovite mica surfaces were modified with glutaraldehyde as described earlier. ${ }^{29}$ Briefly, $300 \mu \mathrm{L}$ of aminopropyltriethoxysilane (Sigma), $100 \mu \mathrm{L} \mathrm{N}, \mathrm{N}$-diisopropylethylamine (Sigma), and freshly cleaved mica sheets were sequentially placed inside a sealed desiccator with $\sim 5 \mathrm{~L}$ volume, and its atmosphere was purged with argon gas. After 3 hours of vapor deposition, mica sheets were removed, and $200 \mu \mathrm{L}$ of $1 \mathrm{mM}$ glutaraldehyde (Sigma) was pipetted onto the modified surface, incubated for 10 minutes at room temperature, washed extensively with MilliQ water and finally with the sample buffer. Typically, $100 \mu \mathrm{L}$ of sample was then added on the surface and subsequently washed with sample buffer after 10 minutes. Immediately before deposition, samples were prepared by diluting stock solutions of WT TTR down to $0.5 \mu \mathrm{g} / \mathrm{mL}$ in $10 \mathrm{mM}$ HEPES buffer, $\mathrm{pH} \mathrm{7}$, and $150 \mathrm{mM} \mathrm{NaCl}$, while acidified amyloid samples were diluted to 1to 
$3 \mu \mathrm{g} / \mathrm{mL}$ in $50 \mathrm{mM}$ sodium acetate buffer at $\mathrm{pH}$ 3.6. The cantilever deflection was calibrated against the surface of freshly cleaved mica, and the spring constant of the cantilever (typically $\sim 30 \mathrm{pN} / \mathrm{nm}$ ) was determined with the thermal method. ${ }^{30}$ After image acquisition, in situ force spectroscopy to promote mechanical unfolding of single molecules was performed by pressing the cantilever tip onto preselected coordinates of the image and moving the cantilever away from the sur-

where $F$ is force, $z$ is extension, $T$ is temperature, $k_{B}$ is the Boltzmann constant, $L_{C}$ is the contour length, and $L_{P}$ is the persistence length. The main criterion used to select force curves was the analysis WLC model constrained to $L_{P}=400 \mathrm{pm}$ to determine the contour length for each force transition. Statistical significance was determined using the Wilcoxon signed-rank test. Error values indicated in the text are standard errors of the mean (s.e.m.). Relevant statistics are tabulated in the Supplementary information section (Table S1).

\section{3 | RESULTS}

Transthyretin amyloid annular oligomers (aoTTR) were observed within the first 24 hours after commencement of aggregation by acidification as reported earlier. ${ }^{19}$ Evidence of their annular morphology was apparent in the height-, amplitude-, and phase-contrast AFM images (Figure 2A). Height-contrast images show topological proper- F2 71 ties similar to our previous report, ${ }^{19}$ notably a diameter of $\sim 15 \mathrm{~nm}$ (Figure 2B,C) and heights of $2.47 \pm 0.09 \mathrm{~nm}$ (Figure 2C,E). These characteristics are markedly different from those obtained on native TTR (nTTR), which was imaged in phosphate buffer saline (Figure 2D) showing the presence of globular particles with heights of $1.03 \pm 0.05 \mathrm{~nm}$ (Figure 2E).

Both nTTR and aoTTR were manipulated by force spectroscopy. The obtained force curves showed marked differences in both the extension of the manipulated chains and in the number of intermediates occurring during pulling (Figure 3A). While in the case of nTTR, F3 81 force curves typically exhibited a single mechanical event corresponding to the detachment of the molecule from the tip, in case of aoTTR we often observed curves containing up to 8 events (Figure 3B). The curves were fitted with the WLC model to reveal the parameters of the manipulated protein chain. The persistence length values were distributed around $0.4 \mathrm{~nm}$ : mean values were $0.491 \pm 0.017 \mathrm{~nm}$ and $0.464 \pm 0.019 \mathrm{~nm}$ for $\mathrm{nTTR}$ and aoTTR, respectively (Figure $3 \mathrm{C}$ ). In of the persistence length whereby curves containing most events with $L_{P}<300 \mathrm{pm}$ were discarded. Selected curves were again fitted with the

FIGURE 2 Atomic force microscopy imaging in liquid. A, Annular oligomers detected by 3 different contrast modalities: height (left), amplitude (center), anđ phase (right). B, Three selected oligomers ềidence circular perimeter and a hollow core: scale bar is $15 \mathrm{~nm}$ and applies to all 3 panels. C, A 3D rendition of the topological map emphasizes annular configuration of oligomers. D, Images of native transthyretin (TTR) dispersed in phosphate buffer saline buffer. E, Histogram of particle heights; nTTR and aoTTR refer to the native and annular oligomeric forms of TTR 
(A)

Native TTR

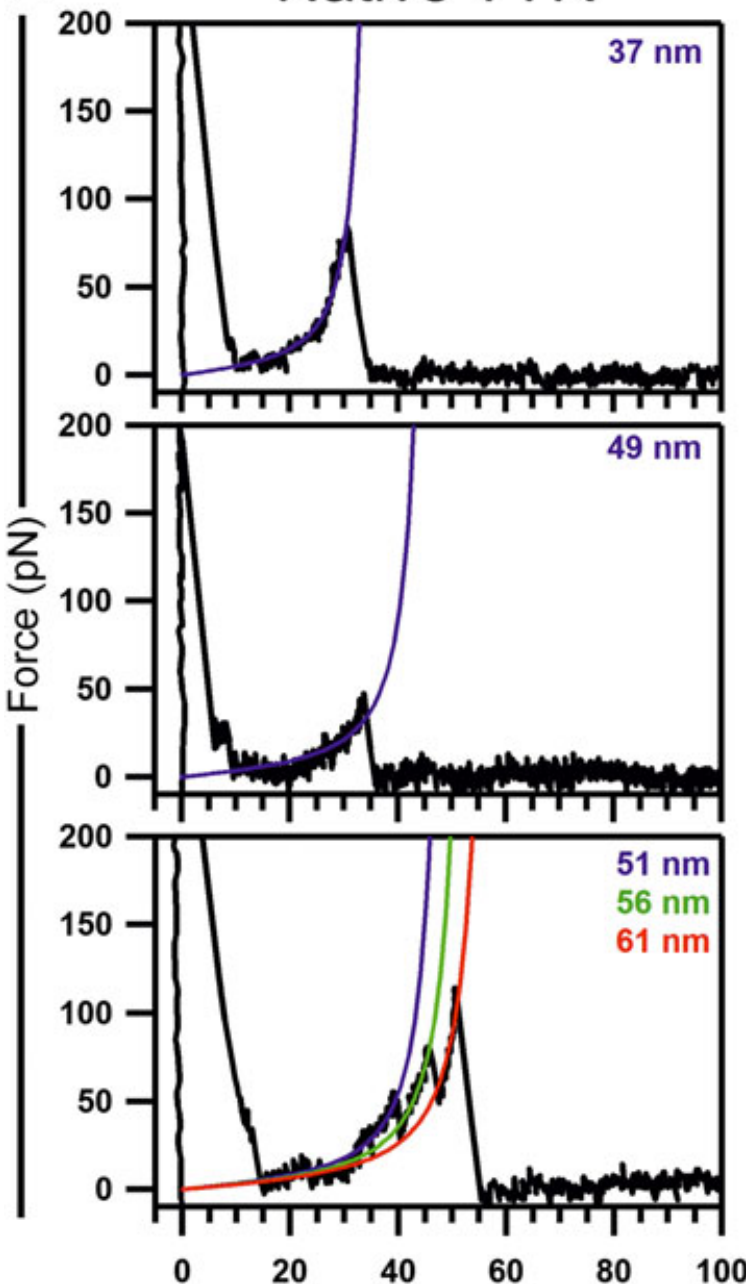

Annular Oligomers
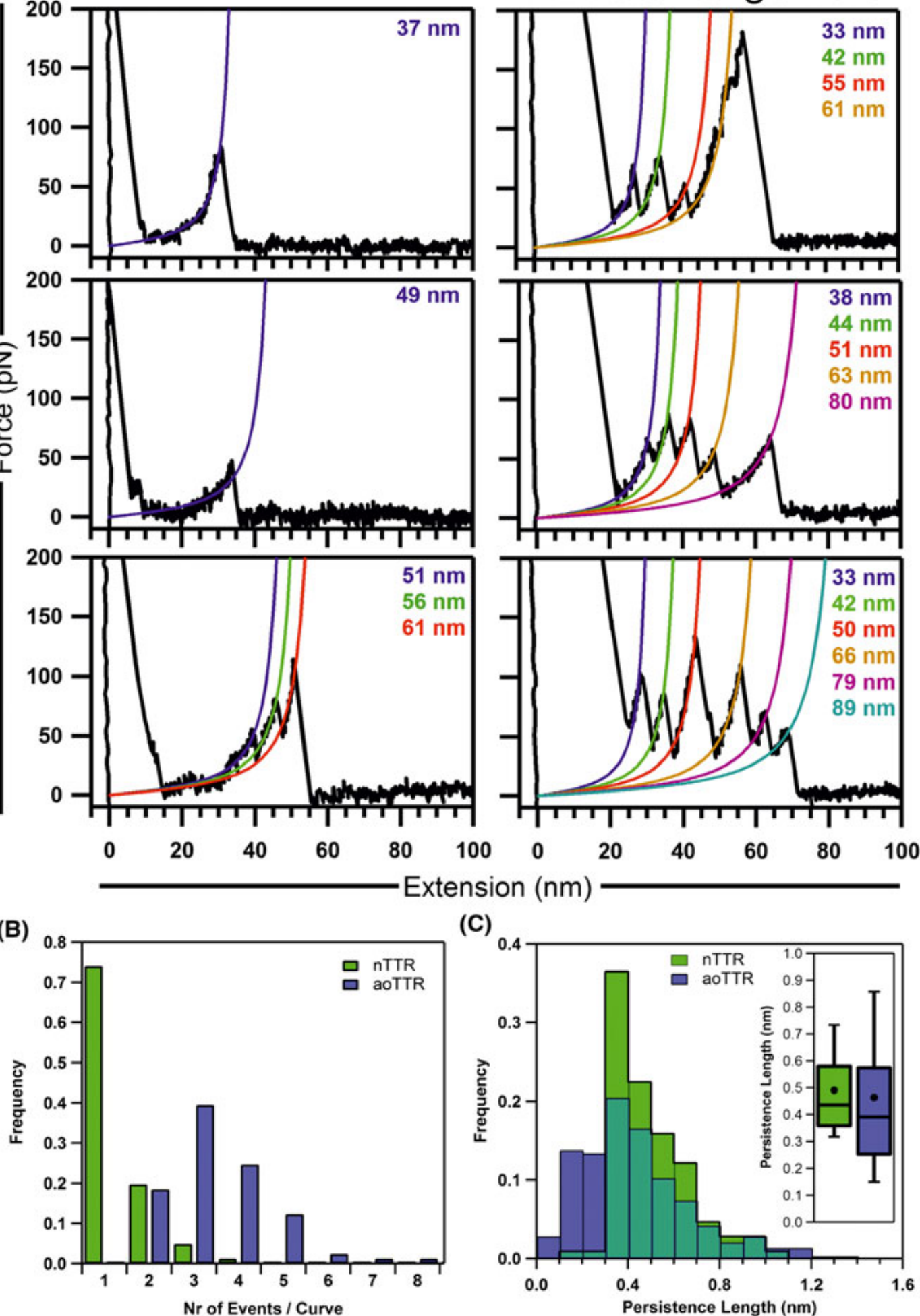

(C)

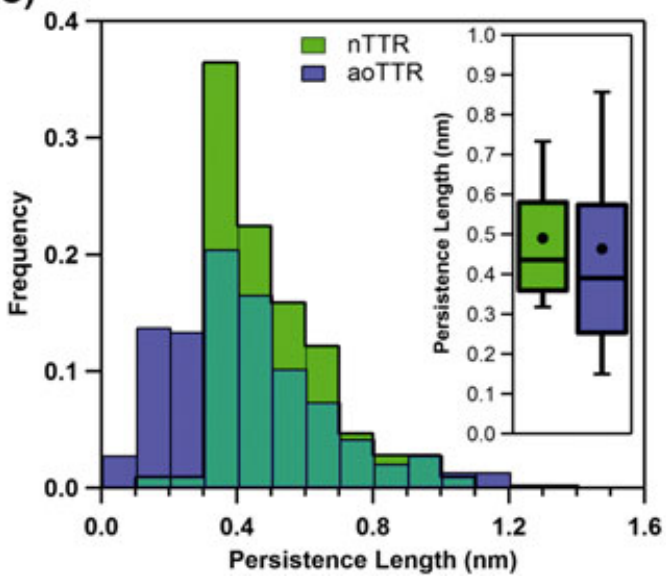

FIGURE 3 Force spectroscopy data. A Comparison of force curves obtained for the native and the amyloid annular oligomeric form of transthyretin (TTR) highlights differences with respect to the frequency of force transitions and total length of the manipulated chains. Colored lines are worm-like chain fits with $L p=0.4 \mathrm{~nm}$, and the resulting $L c$ values are indicated on the right. $B, \mathrm{nTTR}$ typically unfolded in a single step, with 1 event detected per curve, whereas aoTTR curves displayed multiple force peaks. C, Distribution of persistence lengths using unconstrained

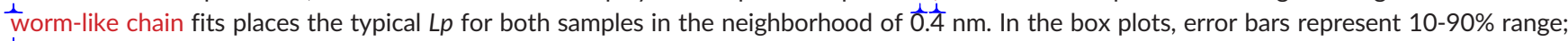
central line and dot correspond to median and mean, respectively

We began by analyzing the $L c$ of the last observed event, or the tour lengths $\left(L_{C}\right)$ associated with the protein chains and the intermedimaximal contour length (LC-Max) corresponding to the detachment of the sample from the tip. While in the case of nTTR a limit of up to 
$\sim 50 \mathrm{~nm}$ was typically attained, this was often approximately the dou-

F4 ble in the case of aoTTR (Figure 4A). Considering that the TTR monomer with its $\mathrm{N}$-terminal signal methionine has 128 residues and therefore a contour length (Lc-Monomer) of $51.2 \mathrm{~nm}$, the results may also be expressed as a fraction of the length of the TTR monomer. This analysis shows (Figure 4A) that when manipulating nTTR, typically we stretched, on average, a fractional size of $0.66 \pm 0.02$ of the TTR monomer. By contrast, for aoTTR the fractional size was on average $1.41 \pm 0.03(P<.001)$. This is also apparent when examining the $L c$ associated with the subsequent unfolding events as a function of their rank within the sequence of the force spectrum (Figure 4B). The subsequent unfolding events typically occurred separated by approximately $10 \mathrm{~nm}$, and up to $\sim 48 \mathrm{~nm}$ for nTTR and up to $\sim 88 \mathrm{~nm}$ for aoTTR. The spacing between intermediate events $(\Delta \mathrm{Lc})$ was analyzed more carefully for aoTTR (Figure 4C). Because the position of the last event is randomly located because it is determined by the site of tip-sample interaction and the moment of detachment, the spacing between the 2 last events was excluded from the analysis. The histogram displays a multimodal distribution where peaks appear at multiples of $4 \mathrm{~nm}$ and up to $18 \mathrm{~nm}$. Typically, transitions occurred separated by $\sim 8 \mathrm{~nm}$, representing $50 \%$ of the analyzed $\Delta \mathrm{Lc}$ values. These intermediate transitions also occurred at increased force for aoTTR when compared
(A)

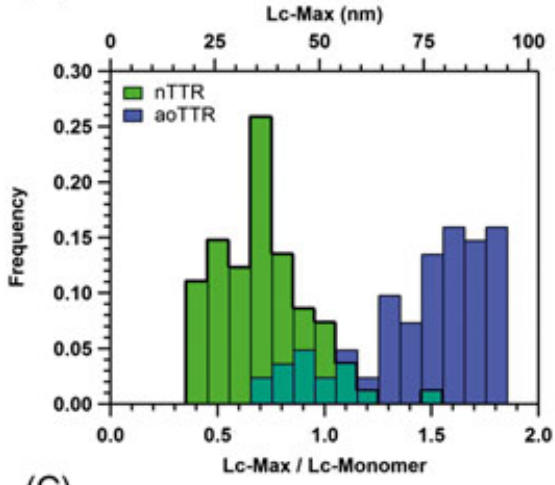

(C)

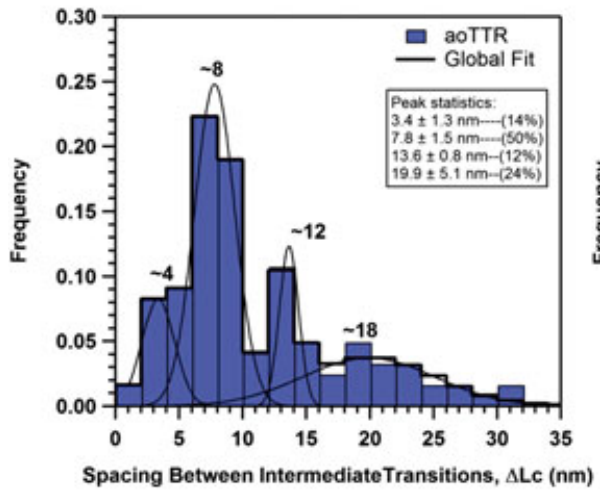

with nTTR (Figure 4D), or $95 \pm 4$ and $57 \pm 10 \mathrm{pN}$, respectively $(P<.001)$.

\section{4 | DISCUSSION}

\subsection{AFM imaging of amyloid annular oligomers}

Amyloid annular oligomers have been imaged by AFM during aggregation of different proteins. ${ }^{19,31-33}$ Compared to other annular assemblies such as ion channels, ${ }^{34}$ the AFM images of amyloid annular oligomers are often more fuzzy, possibly because of on one hand, annular oligomers are unstable complexes and, on the second, because their orientation is likely more random than in the case of membrane proteins. They nonetheless reveal an annular configuration (Figure $2 \mathrm{~A}$ ) that is more striking in the phase-contrast AFM images as seen also earlier. ${ }^{19}$ Topographic mapping further indicates that these oligomeric species have morphological properties similar to those previously identified in the TTR aggregation pathway, ${ }^{19}$ including diameters in the range of $15 \mathrm{~nm}$ (Figure 2B,C) and heights of $\sim 2.5 \mathrm{~nm}$ (Figure 2E). By contrast, images of TTR in phosphate buffer saline display the presence of particles with an average height of $\sim 1 \mathrm{~nm}$. As

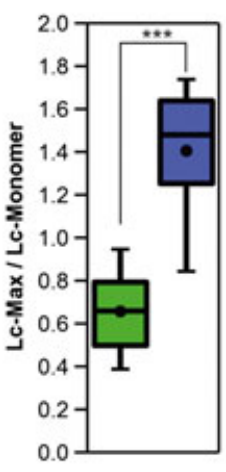

(D)

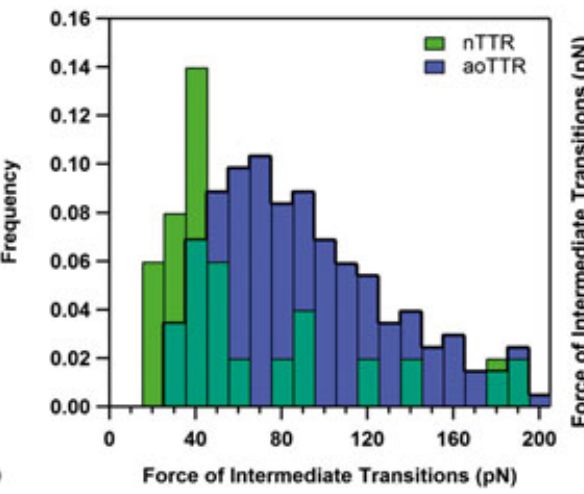

(B)
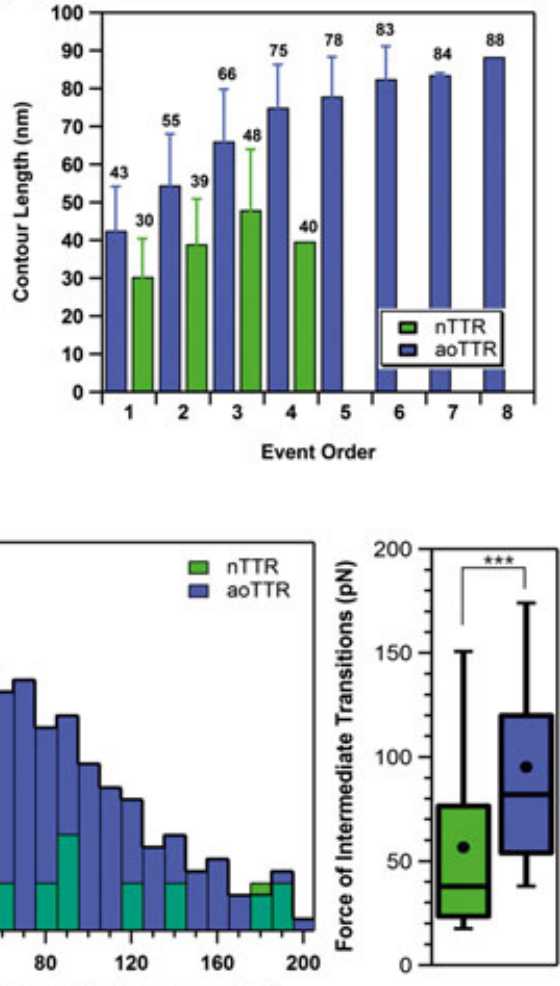

FIGURE 4 Analysis of contour length and force values. A, Evaluation of the maximal contour length (Lc-Max) of the manipulated chains shown in distance units (histogram, top axis) and as a fraction of the contour length of the transthyretin (TTR) monomer (histogram, bottom axis, and boxplot). B, Average contour length as a function of ranked order of unfolding events within a force spectrum shows that increments of $\sim 10 \mathrm{~nm}$ are common. Numbers above bars are the average Lc. The last values of both series are single measurements and therefore contain no error bars. Error bars represent standard deviation. Sample size is 108 for $n T T R$ and 283 aoTTR. C, Histogram of the spacing between consecutive transitions ( $\Delta L C$ ) shows multiples of 4 up to $12 \mathrm{~nm}$, and an additional peak at $18 \mathrm{~nm}$. D, Histogram and box-plot comparison of the force registered for unfolding intermediates. The force of the last event (tip-sample detachment) wâs discarded from this analysis. In the box plots, error bars represent $10-90 \%$ range; central line and dot correspond to median and mean, respectively. Statistical significance at $P<.001$ is denoted by ${ }^{* * *}$ 
reported earlier on the basis of volume calculations derived from AFM data, these particles are likely to represent mostly TTR monomers. ${ }^{19}$ Although the functional TTR is a tetramer, on extreme dilutions such as those required for AFM imaging, the TTR tetramer tends to dissociate into its constituting units even at physiological conditions of $\mathrm{pH}$ and ionic strength. ${ }^{18}$ However, we can also notice some extent of heterogeneity in the particle population (Figure 2D), which may not be so clear

from the height measurements alone (Figure 2F) but becomes more apparent when volume calculations are used. ${ }^{19}$ Thus, nTTR samples appear to be composed mostly of TTR monomers and to a smaller extent also of dimers and tetramers.

\subsection{Persistence length as a selection criterion of force curves}

Manipulation of nTTR and aoTTR resulted in sawtooth-shaped force transitions present at extensions up to $\sim 100 \mathrm{~nm}$ (Figure 3). These force curves revealed transitions that occurred several times in any one pulling of aoTTR, while in the case of nTTR most often only a single event resulting from sample-tip detachment was observed (Figure 2B). Protein unfolding experiments by single molecule force spectroscopy typically involve either the usage of molecular handles through which tension is applied to the system being probed ${ }^{35,36}$ or rely on natural or recombinant systems consisting of a series of concatenated polypeptide modules or polyproteins. ${ }^{36}$ These 2 approaches allow for a clearer isolation of the protein being unfolded, thereby minimizing the interference from the tethering surfaces and limiting the danger of simultaneous manipulation of multiple chains. However, in the context of protein aggregation, the presence of such handles or a system composed by multiple repeats is a poor option, because it would introduce new types of intermolecular interactions that may alter the aggregation process. There is therefore the danger that manipulation of small proteins with their natural composition will contain events that result from the pulling of several chains in parallel. This is particularly true in aggregated species because of the high proximity between different protein molecules.

It has been shown that the pulling of 2 polypeptide chains in parallel results in an apparent halving of $L p$ values with respect to the $0.4 \mathrm{~nm}$ expected for a single polypeptide chain. ${ }^{37}$ We therefore used the criterion of accepting a curve for further analysis if under unconstrained fitting of the WLC the fraction of events with $L p>0.3 \mathrm{~nm}$ would be greater than that of events with $L p<0.3 \mathrm{~nm}$. A $0.1 \mathrm{~nm}$ tolerance in $L p$ with respect to the expected $0.4 \mathrm{~nm}$ was introduced to account for most unfolding events occurred over fairly small length scales $(\sim 10 \hat{\mathrm{nm}})$, and for the impact of pulling geometry over short distances. In both cases, the fitting accuracy can be compromised, and the apparent $L p$ deviates from the expected value. Despite ne upper limit for $L p$ was imposed, the curves thus selected for analysis resulted in average and median $L p$ values that are very close to $0.4 \mathrm{~nm}$ in both nTTR and aoTTR (Figure 2C). In both cases a frequency distribution with a main peak at $0.4 \mathrm{~nm}$ was observed (Figure 2C). For aoTTR the occurrence of force transitions with $\mathrm{Lp}<0.4$ is more pronounced and is likely because of the more condensed state of the sample. Indeed, they appear to have a greater impact for events that occur closer to the surface in the case of aoTTR, but are typically absent in the case of nTTR (Figure S1). Altogether, the values of $L p$ obtained from fits of the WLC model to the transitions observed fall within those reported in the literature, which typically vary between $0.31 \mathrm{~nm}$ for a glycine homopolymer and $0.59 \mathrm{~nm}$ for stiffer polypeptides with high $\psi$ dihedral angle potentials. ${ }^{38}$

\section{3 | Contour length of manipulated chains}

Analysis of the contour length of the final event reveals striking differences between the 2 samples analyzed. It indicates that the manipulated chains in aoTTR are longer than those of nTTR (Figure 4A). Because the TTR monomer is 128 residues long, or $51.2 \mathrm{~nm}$ (considering a $0.4 \mathrm{~nm}$ length for each residue), we can see that while in the case of $\mathrm{nTTR}$ only a fraction of the TTR monomer was manipulated, for aoTTR the manipulated chains are greater than that of a single monomer and likely correspond to the distance of a TTR dimer (Figure 4). The observation in aoTTR of chain lengths that are equivalent to that of a dimer, but not more, is suggestive of a head-to-head and tail-totail orientation of the monomers (Figure 5D). If monomers associated F5 78 in a head-to-tail arrangement, then the monomer-monomer interface would be replicated at every interface, resulting in a polymeric arrangement and hence much longer chains and apparent contour lengths. In fact, earlier studies suggest that head-to-head dimers may serve as basic building blocks for the assembly of TTR amyloid aggregates that may share the same subunit interface as the native dimer. ${ }^{20}$ In that study the authors reported that TTR monomers containing cysteine mutations in the F-strand, resulting in crosslinked head-to-head dimers, produced amyloid aggregates with very similar kinetic profile as the wild-type form of the protein. A follow-up study from the same group further suggested extensive rearrangement of the edge strands $C$ and $D$ that exposed residues of strand $B$ which would hence partake a new amyloid TTR interface. ${ }^{39}$ More recently, the design of peptides that specifically bind to strands F and H of TTR have been shown to effectively work as amyloid inhibitors by capping those strands and preventing head-to-head association of TTR monomers. ${ }^{17}$ The particular relevance of dimers over other similar low molecular weight aggregates (eg, trimers, tetramers, and pentamers) is not exclusive to TTR and has been reported in a variety of amyloidogenic systems such as peptides $A \beta^{40}$ and Sup $35,{ }^{41}$ as well as proteins, including $\alpha$ synuclein ${ }^{42,43}$ and $\beta 2$-microglobulin. ${ }^{44}$ Thus, it is conceivable that the arrangement of monomers along the aoTTR annuli might result in alternating strongly and weekly interacting monomer-monomer interfaces, with the strong interfaces associated with dimer formation and weak interfaces defining the dimer outer limits. Interestingly, recent X-ray crystallography data of annular oligomers formed by a derivative of the amyloidogenic A 1 17-36 peptide also pointed at the presence of 2 distinct types of interfaces. ${ }^{45}$ Here it is important to note the shape of the frequency distribution of Lc-Max for aoTTR that displays a sharp decline prior to reaching the complete contour length of a dimer (Figure 4A). This is indicative of a discontinuity with precise physical boundaries. This boundary is less clear for nTTR as the full contour length of the TTR monomer is approached (Figure 4A). X-ray crystallographic structures show that for native TTR the monomer-monomer interface forming the native dimer is stabilized by hydrogen bonding across the F-strand (Figure 1). Our AFM images of nTTR (Figure 2D) 
(A)

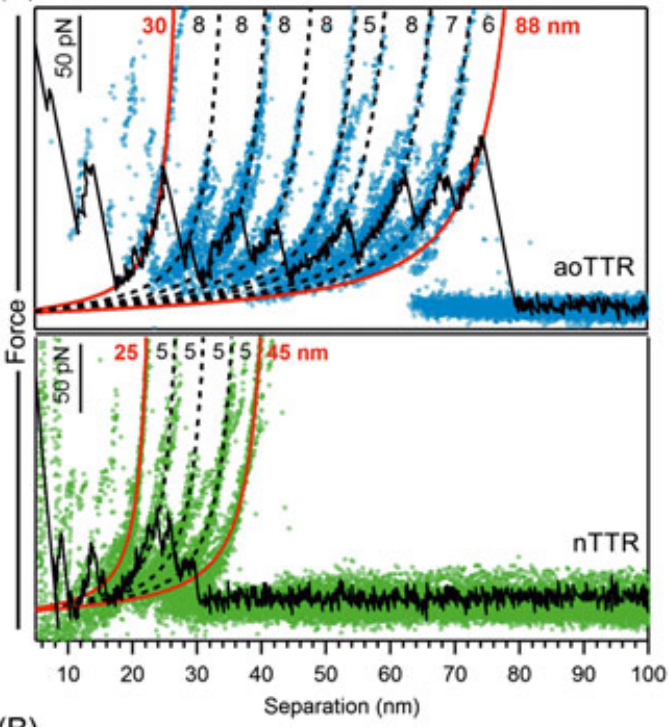

(B)

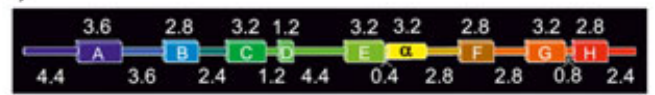

(C)

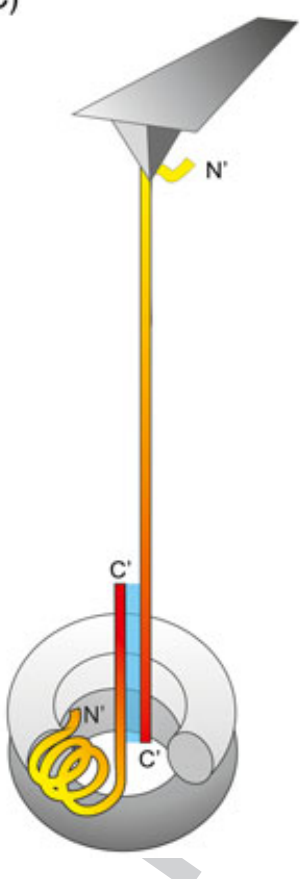

(D)

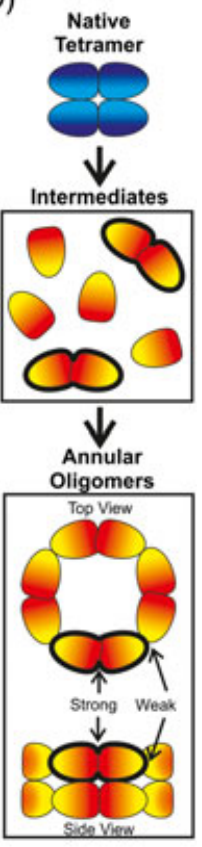

FIGURE 5 Mechanical unfolding and secondary structure of transthyretin (TTR). A, Overlay of different curves from the manipulation of aoTTR (above) and nTTR (below). B, Secondary structure of TTR (PDB entry 2PAB) measured in nanometers (numbers) and where letters (A-H) indicate $\beta$-strands and a represents the sole $\alpha$-helix; colors are as represented in Figure 1. C, Schematic representation of the pulling of a dimer from aoTTR where dimerization is likely to occur through participation of residues toward the ' $C^{\prime}$ terminus. The double-stack arrangement of aoTTR likely contributes to stabilize unfolding intermediates. D, Diagram of the assembly of annular oligomers with monomers oriented in a head-to-head and tail-to-tail configuration forming strong and weakkly interacting interfaces

suggest that a mixture of both monomers and dimers is likely to be present. Therefore, while for nTTR the chain length of the TTR monomer does not represent an insurmountable limit for Lc-Max values, this limit appears to be more defined at the level of the dimer for aoTTR. In addition, the high frequency of pullings observed for chains that are up to $\sim 1.8 \times$ the length of the TTR monomer (Figure $4 \mathrm{~A}$ ) is also suggestive that the interface might occur at the termini of the monomer sequence. This corroborates findings in previous reports already discussed here, where the monomer-monomer interface of the amyloidogenic dimer likely involves residues of the edge strands that lie toward the C-terminus of the sequence. ${ }^{17,20,39}$ In fact, for aoTTR we observe pullings that on average are $1.41 \times$ the monomer contour length (or $\sim 72 \mathrm{~nm}$ ), which is remarkably close to a structure of $2 \mathrm{sym}$ metrically oriented TTR monomers overlapping at their F-strands, which would result in a structure $1.46 \times$ the size of a monomer (or $\sim 75 \mathrm{~nm}$ long). We observe curves that can be even longer is indicative that residues in the F-strand are not the only ones participating in the interface, and as proposed earlier, ${ }^{17}$ additional residues further downstream of the $\hat{F}$-strand are also involved in forming the internal dimer interface (Figure $5 \mathrm{C}$ ).

\subsection{Contour length of unfolding intermediates}

Pulling on aoTTR revealed a series of unfolding intermediates (Figure 3A). Typically, 2 intermediates were observed, corresponding to 3 events per force-extension curve (Figure 3B). In fact, we did not observe any curves in aoTTR that passed our selection criterion and that would contain no intermediates. This is in striking contrast with the nTTR where $\sim 70 \%$ of curves showed the unfolding of the manipulated chain in a single step (Figure 3B). The immunoglobulin domain I27 of the sarcomeric protein Titin, which has been extensively used in force spectroscopy experiments, ${ }^{46}$ typically unfolds through overcoming a single energy barrier. ${ }^{46,47}$ Like the TTR monomer, the I27 domain is a $\beta$-sheet protein containing 8 -strands forming 2 betasheets that stack on in each other to form a $\beta$-sandwich fold, and revealing a so called "Greek key" topological motif. Interestingly, molecular dynamic simulations reveal that the 127 dimer, rather than the trimer or the tetramer, is the smallest unit that will spontaneously misfold. ${ }^{48}$ Differences in the extent with which unfolding intermediates are observed in aoTTR in comparison to nTTR are suggestive of considerable folding differences, and also of stabilization of unfolding intermediates of the aoTTR dimer by its neighboring environment. To illustrate this last point, we may take the example of 2 different classes of a-helical proteins: while membrane-embedded $\hat{G}$-protein coupled receptors tend to unfold via the stepwise unraveling of their individual transmembrane a-helices, ${ }^{49}$ soluble $a$-helical bundles of spectrin unfold in a single event. ${ }^{50}$ Likewise, mechanical unfolding of $\beta$-barrel membrane proteins such as OmpG reveals multiple unfolding intermediates while the water-soluble $\beta$-barrel GFP does not. ${ }^{51}$ Thus, the molecular environment in which a protein is found during unfolding has a strong impact in defining the roughness of a protein's folding energy landscape. This is believed to result from as proteins unfold in solution, water molecules begin to destabilize its hydrophobic core, thereby smoothing (and also lowering) the energy profiles leading to 
the unfolded state..$^{51}$ In a membrane, however, the presence of lipid molecules will shield hydrophobic regions from the solvent. As each transmembrane region is loaded and stretched out, the hydrophobic core and hydrophilic lipid headgroups will stabilize the remaining sections of the protein still inserted in the membrane bilayer, thus providing steep energy barriers to the unfolding of intermediates. ${ }^{51}$ Therefore, it is conceivable that the unfolding intermediates observed during pulling of aoTTR derive from the stability provided by contacts between dimers within the annular assembly. It has long been recognized that in the general case of protein aggregation, and of amyloid formation in particular, hydrophobic interactions play a key role in protein condensation. In this regard, TTR aggregation is no different. ${ }^{52}$ Furthermore, the importance of a yet unknown network of hydrogen bonding that may be formed across dimers may also be relevant. Previous detailed topographic characterization of aoTTRs indicates that these oligomers assemble to form a stack of 2 annuli, as suggested by a bimodal distribution of their heights with peaks $\mathrm{a} \sim 1 \mathrm{~nm}$ for one annulus, and $\sim 2 \mathrm{~nm}$ for 2 annuli. ${ }^{13}$ Here, given their average height of $2.45 \mathrm{~nm}$ (Figure 2E), annular oligomers appear to predominantly belong to the second population, formed of stacked annuli. Thus, it is conceivable that it was in a context where dimers establish contacts both along the axis of revolution of the annuli and between its 2 layers, that the unfolding of aoTTR dimers took place (Figure $5 \mathrm{C}$ ).

Analysis of the average contour length as a function of the ranked order of the unfolding events indicates that transitions appear to be typically separated by $\sim 10 \mathrm{~nm}$ with standard deviations in the same range (Figure 4B). Using a curve containing the largest number of events as a reference, and by offsetting several curves by $\pm 10 \mathrm{~nm}$ with respect to the reference curve, we were able to overlay several force spectra that highlight a fairly regular unfolding pattern in aoTTR dimers (Figure 5A). This pattern shows transitions that are separated by $\sim 8 \mathrm{~nm}$, consistent with the frequency distribution of $\Delta \mathrm{Lc}$ values where a predominant peak at $\sim 8 \mathrm{~nm}$ is observed (Figure $4 \mathrm{C}$ ) and closely matching the increment in contour length observed in Figure 4B. Such unfolding pattern closely replicated in different pulling experiments underscores the highly organized structure of the system being probed. It suggests that the underlying organization and structure of a given molecule can be observed in a recurrent fashion, in different molecules. The $\Delta L c$ distribution also suggests the presence of a fundamental mode at $\sim 4 \mathrm{~nm}$, and additional modes at $\sim 12$ and $\sim 18 \mathrm{~nm}$. Considering the contour length of the secondary structure elements in TTR, these values are compatible with the sequential unfolding of $\beta$-strands (Figure $5 \mathrm{~B}$ ). Because the exact structure of TTR within amyloid aggregates is currently unknown, it is at this stage speculative to infer whether these transitions represent the peeling of individual large $\beta$-strands or blocks of smaller $\beta$-strands such as a hairpin of 2 consecutive $\beta$-strands. However, several reports have highlighted that the structure of TTR within these aggregates might share many similarities with the native conformation of the TTR monomer. The native TTR monomer comprises a series of $8 \beta$-strands and $1 \alpha$-helix joined by 8 interconnecting segments and 2 terminal regions (Figure $5 B$ ). On average, each of these regions has a length of $2.7 \mathrm{~nm}$, which would mean that an unfolding step with a length $\sim 8 \mathrm{~nm}$ would correspond to the extension of 3 of these sections $(8.1 \mathrm{~nm})$. It could, for example,

correspond to the stretching and unfolding of $\beta$-hairpin structure. Regardless, an extensive dimer-dimer interface will have to exist along the outer surface of at least one of the $\beta$-sheets promoting the stabilization of its $\beta$-strands. This type of dimer-dimer interface will likely be very different from that found in the tetrameric arrangement of the 2 native TTR dimers, which relies on very weak interactions that need to be stabilized to mitigate conversion of TTR into its amyloidogenic form.

\section{5 | Unfolding force of intermediates}

Manipulation of aoTTR resulted in the emergence of intermediates whose mechanical transitions occurred at higher force values than those observed in nTTR (Figure 4D). This difference could be interpreted because of amyloid oligomers typically attain a more stable state than the native protein from which they are derived. ${ }^{53-61}$ However, such interpretation should be viewed with some caution. On one hand, we are comparing the unfolding forces of intermediates, which emerged more rarely in the case of nTTR. For nTTR, most of the force values were discarded from the analysis as these correspond to curves that contained no intermediates, and the force of those transitions is ultimately because of the tip-sample interaction. On the other hand, mechanical strength of proteins may not necessarily correlate with their thermodynamic stability. ${ }^{62}$ It is viewed that prefibrillar precursors sample distinct conformations as they travel along a rough energy landscape toward more stable conformations. ${ }^{63}$ However, along this process they may become kinetically trapped ${ }^{64}$ in conformations that result in what are called "off-pathway" oligomers, ${ }^{65-67}$ which may or may not be necessarily more stable than the native conformation. ${ }^{68}$ Little is known about the stability of annular oligomers and of the dimers found within them. Although they may have crossed an energy barrier that may lead to a more stable arrangement, at this stage the aoTTR dimer may still not represent a more stable conformation when compared to nTTR. An increase in force may be explained by a variety of reasons, which with our approach may be difficult to scrutinize. For example, as highlighted in the previous paragraphs, manipulation of aoTTR dimer occurred in a context where intermolecular interactions between neighboring dimers, and differences in buffer composition can play an important role. Because AFM imaging suggests that nTTR was mostly present in the monomeric form (Figure 2D,E), differences in the molecular context of the manipulated chains limit our ability to compare force values. As discussed earlier, the importance of molecular context can be relevant in the definition of the roughness of the energy landscape. In addition, the pulling geometry can affect force as shown earlier for the unfolding of a $\beta$-sheet protein. ${ }^{\frac{6}{99}}$ Given the annular arrangement of oligomers, it is conceivable that they are organized in a preferential orientation with respect to the pulling axis, rather than randomly distributed, which we anticipate to be more likely in the case of nTTR molecules. If so, a preferential orientation with respect to the pulling direction may emphasize one particular unfolding trajectory for which higher forces would be required, while for the case of nTTR these trajectories would be more widely sampled and thus averaged out. 


\section{5 | CONCLUSION}

We demonstrate using single-molecule force spectroscopy that native TTR and annular oligomers exhibit distinct unfolding patterns. While $\mathrm{nTTR}$ tends to retain a monomeric structure and unfolds in 1 step, aoTTR unfolds via a series of intermediate structures across a length range equivalent to the contour length of a concatenated TTR dimer.

These intermediate structures display a contour-length increment of $\sim 8 \mathrm{~nm}$, which corresponds to a section of protein chain likely stabilized by intermolecular contacts established by aoTTR dimers within the annular assembly. Monomers arranged in a head-to-head orientation may form an interface that shares some structural similarities with the native dimer. However, when compared with the native tetrameric molecule, dimers in aoTTR are likely to interact with other dimers in a very different way, notably through extensive contacts along their $\beta$-strands.

In general, we have shown that through careful selection of force curves using persistence length as a criterion, we are able to select data that can be interpreted and rationalized in agreement with data available for the aggregation pathway of TTR. Because this approach requires no handles, it may open the door to the analysis of other systems, which, like ours, may become distorted when analyzed in tandem repeats, or in the presence of molecular handles. In addition, transient structures that may be too dynamic or heterogeneous to be captured by higher resolution methods may, in a similar way, be probed and understood with greater structural detail.

\section{ACKNOWLEDGMENTS}

This work was supported by grants from the Hungarian Science Foundation (OTKA K109480) and the National Research, Development and Innovation Office under the contract VKSZ_14-1-2015-052. The research leading to these results has received funding from the European Union's Seventh Framework Program (FP7/2007-2013) under grant agreement no HEALTH-F2-2011-278850 (INMiND).

\section{REFERENCES}

1. Alshehri B, D'Souza DG, Lee JY, Petratos S, Richardson SJ. The diversity of mechanisms influenced by transthyretin in neurobiology: development, disease and endocrine disruption. I Neuroendocrinol. 2015;27(5):303-323. doi: 10.1111/jne.12271

2. Maleszewski JJ. Cardiac amyloidosis: pathology, nomenclature, and typing. Cardiovasc Pathol. 2015;24(6):343-350. doi: 10.1016/j. carpath.2015.07.008

3. Wechalekar AD, Gillmore JD, Hawkins PN. Systemic amyloidosis. Lancet. 2015. doi: 10.1016/S0140-6736(15)01274-X

4. Robbins J. Transthyretin from discovery to now. Clin Chem Lab Med. 2002;40(12):1183-1190. doi: 10.1515/CCLM.2002.208

5. Damas AM, Saraiva MJ. Review: TTR amyloidosis-structural features leading to protein aggregation and their implications on therapeutic strategies. J Struct Biol. 2000;130(2-3):290-299. doi: 10.1006/ jsbi.2000.4273

6. Johnson SM, Wiseman RL, Sekijima Y, Green NS, Adamski-Werner SL, Kelly JW. Native state kinetic stabilization as a strategy to ameliorate protein misfolding diseases: a focus on the transthyretin amyloidoses. Acc Chem Res. 2005;38(12):911-921.

7. Nakagawa M, Sekijima Y, Yazaki M, et al. Carpal tunnel syndrome: a common initial symptom of systemic wild-type ATTR (ATTRwt) amyloidosis. Amyloid. 2016;23(1):58-63. doi: 10.3109/ 13506129.2015.1135792

8. Nagasaka T. Familial amyloidotic polyneuropathy and transthyretin. Subcell Biochem. 2012;65:565-607. doi: 10.1007/978-94-007-54164_21

9. Lai Z, Colon W, Kelly JW. The acid-mediated denaturation pathway of transthyretin yields a conformational intermediate that can self-assemble into amyloid. Biochemistry. 1996;35(20):6470-6482. doi: 10.1021/ bi952501g

10. Naiki H, Okoshi T, Ozawa D, Yamaguchi I, Hasegawa K. Molecular pathogenesis of human amyloidosis: lessons from beta microglobulin-related amyloidosis. Pathol Int. 2016. doi: 10.1111/ pin.12394

11. Nixon RA, Cataldo AM. Lysosomal system pathways: genes to neurodegeneration in Alzheimer's disease. J Alzheimers Dis. 2006;9(3 Suppl):277-289.

12. Cardoso I, Goldsbury CS, Muller SA, et al. Transthyretin fibrillogenesis entails the assembly of monomers: a molecular model for in vitro assembled transthyretin amyloid-like fibrils. J Mol Biol. 2002;317(5):683-695. doi: 10.1006/jmbi.2002.5441

13. Pires RH, Saraiva MJ, Damas AM, Kellermayer MS. Structure and assembly-disassembly properties of wild-type transthyretin amyloid protofibrils observed with atomic force microscopy. J Mol Recognit. 2011;24(3):467-476. doi: 10.1002/jmr.1112

14. Faria TQ, Almeida ZL, Cruz PF, Jesus CS, Castanheira P, Brito RM. A look into amyloid formation by transthyretin: aggregation pathway and a novel kinetic model. Phys Chem Chem Phys. 2015;17(11):72557263. doi: $10.1039 / c 4 c p 04549 a$

15. Annamalai K, Guhrs KH, Koehler R, et al. Polymorphism of amyloid fibrils in vivo. Angew Chem Int Ed Engl. 2016. doi: 10.1002/ anie.201511524

16. Groenning M, Campos RI, Hirschberg D, Hammarstrom P, Vestergaard B. Considerably unfolded transthyretin monomers preceed and exchange with dynamically structured amyloid protofibrils. Sci Rep. 2015;5:11443. doi: 10.1038/srep11443

17. Saelices L, Johnson LM, Liang WY, et al. Uncovering the mechanism of aggregation of human transthyretin. J Biol Chem. 2015;290(48):2893228943. doi: 10.1074/jbc.M115.659912

18. Quintas A, Saraiva MJ, Brito RM. The amyloidogenic potential of transthyretin variants correlates with their tendency to aggregate in solution. FEBS Lett. 1997;418(3):297-300.

19. Pires RH, Karsai A, Saraiva MJ, Damas AM, Kellermayer MS. Distinct annular oligomers captured along the assembly and disassembly pathways of transthyretin amyloid protofibrils. PLoS One. 2012;7(9) e44992. doi: 10.1371/journal.pone.0044992

20. Serag AA, Altenbach C, Gingery M, Hubbell WL, Yeates TO. Identification of a subunit interface in transthyretin amyloid fibrils: evidence for self-assembly from oligomeric building blocks. Biochemistry. 2001;40(31):9089-9096.

21. Mulaj M, Foley J, Muschol M. Amyloid oligomers and protofibrils, but not filaments, self-replicate from native lysozyme. J Am Chem Soc. 2014;136(25):8947-8956. doi: 10.1021/ja502529m

22. Yagi H, Abe Y, Takayanagi N, Goto Y. Elongation of amyloid fibrils through lateral binding of monomers revealed by total internal reflection fluorescence microscopy. Biochim Biophys Acta. 2014;1844(10):1881-1888. doi: 10.1016/j.bbapap.2014.06.014

23. Diomede L, Di Fede G, Romeo M, et al. Expression of A2 V-mutated Abeta in Caenorhabditis elegans results in oligomer formation and toxicity. Neurobiol Dis. 2014;62:521-532. doi: 10.1016/j.nbd.2013.10.024

24. Harte NP, Klyubin I, McCarthy EK, et al. Amyloid oligomers and mature fibrils prepared from an innocuous protein cause diverging cellular death mechanisms. J Biol Chem. 2015;290(47):28343-28352. doi: 10.1074/jbc.M115.676072 
25. Cecchi C, Stefani M. The amyloid-cell membrane system. The interplay between the biophysical features of oligomers/fibrils and cell membrane defines amyloid toxicity. Biophys Chem. 2013;182:30-43.

26. Capone R, Quiroz FG, Prangkio P, et al. Amyloid-beta-induced ion flux in artificial lipid bilayers and neuronal cells: resolving a controversy. Neurotox Res. 2009;16(1):1-13.

27. Mirzabekov TA, Lin MC, Kagan BL. Pore formation by the cytotoxic islet amyloid peptide amylin. J Biol Chem. 1996;271(4):1988-1992.

28. Almeida MR, Damas AM, Lans MC, Brouwer A, Saraiva MJ. Thyroxine binding to transthyretin Met 119. Comparative studies of different heterozygotic carriers and structural analysis. Endocrine. 1997;6(3):309-315. doi: 10.1007/BF02820508

29. Wang H, Bash R, Yodh JG, Hager GL, Lohr D, Lindsay SM. Glutaraldehyde modified mica: a new surface for atomic force microscopy of chromatin. Biophys J. 2002;83(6):3619-3625. doi: 10.1016/S00063495(02)75362-9

30. Hutter JL, Bechhoefer J. Calibration of atomic-force microscope tips. Rev Sci Instrum. 1993;64(7):1868-1873.

31. Lasagna-Reeves CA, Sengupta U, Castillo-Carranza D, et al. The formation of tau pore-like structures is prevalent and cell specific: possible implications for the disease phenotypes. Acta Neuropathol Commun. 2014;2:56. doi: 10.1186/2051-5960-2-56

32. Quist A, Doudevski I, Lin H, et al. Amyloid ion channels: a common structural link for protein-misfolding disease. Proc Natl Acad Sci U S A. 2005;102(30):10427-10432. doi: 10.1073/pnas.0502066102

33. Sbrana F, Bongini L, Cappugi G, et al. Atomic force microscopy images suggest aggregation mechanism in cerato-platanin. Eur Biophys J. 2007;36(7):727-732. doi: 10.1007/s00249-007-0159-x

34. Muller DJ, Engel A. Atomic force microscopy and spectroscopy of native membrane proteins. Nat Protoc. 2007;2(9):2191-2197. doi: 10.1038/nprot.2007.309

35. Cecconi C, Shank EA, Marqusee S, Bustamante C. DNA molecular handles for single-molecule protein-folding studies by optical tweezers. Methods Mol Biol. 2011;749:255-271. doi: 10.1007/978-1-61779142-0 18

36. Ott W, Jobst MA, Schoeler C, Gaub HE, Nash MA. Single-molecule force spectroscopy on polyproteins and receptor-ligand complexes: the current toolbox. J Struct Biol. 2016. doi: 10.1016/j.jsb.2016.02.011

37. Sarkar A, Caamano S, Fernandez JM. The mechanical fingerprint of a parallel polyprotein dimer. Biophys J. 2007;92(4):L36-L38. doi: 10.1529/biophysj.106.097741

38. Stirnemann G, Giganti D, Fernandez JM, Berne BJ. Elasticity, structure, and relaxation of extended proteins under force. Proc Natl Acad Sci U S A. 2013;110(10):3847-3852. doi: 10.1073/pnas.1300596110

39. Serag AA, Altenbach C, Gingery M, Hubbell WL, Yeates TO. Arrangement of subunits and ordering of beta-strands in an amyloid sheet. Nat Struct Biol. 2002;9(10):734-739. doi: 10.1038/nsb838

40. Lopez del Amo JM, Schmidt M, Fink U, Dasari M, Fandrich M, Reif B. An asymmetric dimer as the basic subunit in Alzheimer's disease amyloid beta fibrils. Angew Chem Int Ed Engl. 2012;51(25):6136-6139. doi: 10.1002/anie.201200965

41. Zhang Z, Chen H, Bai H, Lai L. Molecular dynamics simulations on the oligomer-formation process of the GNNQQNY peptide from yeast prion protein Sup35. Biophys J. 2007;93(5):1484-1492. doi: 10.1529/ biophysj.106.100537

42. Lv Z, Krasnoslobodtsev AV, Zhang Y, et al. Effect of acidic pH on the stability of alpha-synuclein dimers. Biopolymers. 2016. doi: 10.1002/ bip. 22874

43. Marmolino D, Foerch P, Atienzar FA, Staelens L, Michel A, Scheller D. Alpha synuclein dimers and oligomers are increased in overexpressing conditions in vitro and in vivo. Mol Cell Neurosci. 2016;71:92-101. doi: 10.1016/j.mcn.2015.12.012

44. Mendoza VL, Antwi K, Baron-Rodriguez MA, Blanco C, Vachet RW. Structure of the preamyloid dimer of beta-2-microglobulin from

covalent labeling and mass spectrometry. Biochemistry. 2010;49(7):1522-1532. doi: 10.1021/bi901748h

45. Kreutzer AG, Hamza IL, Spencer RK, Nowick JS. X-ray crystallographic structures of a trimer, dodecamer, and annular pore formed by an abeta17-36 beta-hairpin. J Am Chem Soc. 2016. doi: 10.1021/ jacs.6b01332

46. Hoffmann T, Dougan L. Single molecule force spectroscopy using polyproteins. Chem Soc Rev. 2012;41(14):4781-4796.

47. Fowler SB, Best RB, Toca Herrera JL, et al. Mechanical unfolding of a titin Ig domain: structure of unfolding intermediate revealed by combining AFM, molecular dynamics simulations, NMR and protein engineering. J Mol Biol. 2002;322(4):841-849.

48. Zheng W, Schafer NP, Wolynes PG. Free energy landscapes for initiation and branching of protein aggregation. Proc Natl Acad Sci U S A. 2013;110(51):20515-20520. doi: 10.1073/pnas.1320483110

49. Zocher M, Bippes CA, Zhang C, Muller DJ. Single-molecule force spectroscopy of G-protein-coupled receptors. Chem Soc Rev. 2013;42(19):7801-7815. doi: 10.1039/c3cs60085h

50. Law R, Carl P, Harper S, Dalhaimer P, Speicher DW, Discher DE. Cooperativity in forced unfolding of tandem spectrin repeats. Biophys J. 2003;84(1):533-544. doi: 10.1016/S0006-3495(03)74872-3

51. Hensen U, Muller DJ. Mechanistic explanation of different unfolding behaviors observed for transmembrane and soluble beta-barrel proteins. Structure. 2013;21(8):1317-1324. doi: 10.1016/j. str.2013.06.001

52. Sant'Anna R, Braga C, Varejao N, et al. The importance of a gatekeeper residue on the aggregation of transthyretin: implications for transthyretin-related amyloidoses. J Biol Chem. 2014;289(41):2832428337. doi: 10.1074/jbc.M114.563981

53. Berhanu WM, Hansmann UH. Stability of amyloid oligomers. Adv Protein Chem Struct Biol. 2014;96:113-141. doi: 10.1016/bs. apcsb.2014.06.006

54. Berhanu WM, Masunov AE. Can molecular dynamics simulations assist in design of specific inhibitors and imaging agents of amyloid aggregation? Structure, stability and free energy predictions for amyloid oligomers of VQIVYK, MVGGVV and LYQLEN. I Mol Model. 2011;17(10):2423-2442. doi: 10.1007/s00894-010-0912-4

55. Blinov N, Dorosh L, Wishart D, Kovalenko A. Association thermodynamics and conformational stability of beta-sheet amyloid beta(1742) oligomers: effects of $E 22 Q$ (Dutch) mutation and charge neutralization. Biophys J. 2010;98(2):282-296. doi: 10.1016/j.bpj.2009.09.062

56. Chang LK, Zhao JH, Liu HL, et al. Molecular dynamics simulations to investigate the structural stability and aggregation behavior of the GGVVIA oligomers derived from amyloid beta peptide. J Biomol Struct Dyn. 2009;26(6):731-740. doi: 10.1080/07391102.2009.10507285

57. De Simone A, Esposito L, Pedone C, Vitagliano L. Insights into stability and toxicity of amyloid-like oligomers by replica exchange molecular dynamics analyses. Biophys J. 2008;95(4):1965-1973. doi: 10.1529/ biophysj.108.129213

58. Kahler A, Sticht H, Horn AH. Conformational stability of fibrillar amyloid-beta oligomers via protofilament pair formation-a systematic computational study. PLoS One. 2013;8(7) e70521. doi: 10.1371/journal.pone.0070521

59. Li Y, Ji C, Xu W, Zhang JZ. Dynamical stability and assembly cooperativity of beta-sheet amyloid oligomers-effect of polarization. J Phys Chem B. 2012;116(45):13368-13373. doi: 10.1021/jp3086599

60. Porrini M, Zachariae U, Barran PE, MacPhee CE. Effect of protonation state on the stability of amyloid oligomers assembled from TTR(105115). J. Phys Chem Lett. 2013;4(8):1233-1238. doi: 10.1021/ jz400372u

61. Socher E, Sticht H, Horn AH. The conformational stability of nonfibrillar amyloid-beta peptide oligomers critically depends on the C-terminal peptide length. ACS Chem Nerosci. 2014;5(3):161-167. doi: 10.1021/ cn400208r 
62. Oberhauser AF, Badilla-Fernandez C, Carrion-Vazquez M, Fernandez $\mathrm{JM}$. The mechanical hierarchies of fibronectin observed with singlemolecule AFM. J Mol Biol. 2002;319(2):433-447. doi: 10.1016/ S0022-2836(02)00306-6

63. Gershenson A, Gierasch LM, Pastore A, Radford SE. Energy landscapes of functional proteins are inherently risky. Nat Chem Biol. 2014;10(11):884-891. doi: 10.1038/nchembio.1670

64. Hagan MF, Elrad OM, Jack RL. Mechanisms of kinetic trapping in selfassembly and phase transformation. J Chem Phys. 2011;135(10):104115. doi: 10.1063/1.3635775

65. Bellesia G, Shea JE. Diversity of kinetic pathways in amyloid fibril formation. J Chem Phys. 2009;131(11):111102. doi: 10.1063/1.3216103

66. Ma B, Nussinov R. Polymorphic C-terminal beta-sheet interactions determine the formation of fibril or amyloid beta-derived diffusible ligand-like globulomer for the Alzheimer Abeta42 dodecamer. J Biol Chem. 2010;285(47):37102-37110. doi: 10.1074/jbc.M110.133488

67. Uversky VN. Mysterious oligomerization of the amyloidogenic proteins. FEBS J. 2010;277(14):2940-2953. doi: 10.1111/j.1742 4658.2010.07721.x
68. Brummitt RK, Andrews JM, Jordan JL, Fernandez EJ, Roberts CJ. Thermodynamics of amyloid dissociation provide insights into aggregate stability regimes. Biophys Chem. 2012;168-169:10-18. doi: 10.1016/j. bpc.2012.06.001

69. Brockwell DJ, Paci E, Zinober RC, et al. Pulling geometry defines the mechanical resistance of a beta-sheet protein. Nat Struct Biol. 2003;10(9):731-737. doi: 10.1038/nsb968

\section{SUPPORTING INFORMATION}

Additional Supporting Information may be found online in the supporting information tab for this article.

How to cite this article: Pires, R. H., Saraiva, M. J., Damas, A. M., and Kellermayer, M. S. Z. (2016), Force spectroscopy reveals the presence of structurally modified dimers in transthyretin amyloid annular oligomers, J Mol Recognit, doi: 10.1002/jmr.2587 


\section{Journal: Journal of Molecular Recognition}

\section{Article: jmr_2587}

Dear Author,

During the copyediting of your paper, the following queries arose. Please respond to these by annotating your proofs with the necessary changes/additions.

- If you intend to annotate your proof electronically, please refer to the E-annotation guidelines.

- If you intend to annotate your proof by means of hard-copy mark-up, please use the standard proofing marks. If manually writing corrections on your proof and returning it by fax, do not write too close to the edge of the paper. Please remember that illegible mark-ups may delay publication.

Whether you opt for hard-copy or electronic annotation of your proofs, we recommend that you provide additional clarification of answers to queries by entering your answers on the query sheet, in addition to the text mark-up.

\begin{tabular}{|c|c|c|}
\hline Query No. & Query & Remark \\
\hline Q1 & $\begin{array}{l}\text { AUTHOR: Please confirm that given names (red) and surnames/family names (green) } \\
\text { have been identified correctly. }\end{array}$ & \multirow{3}{*}{$\begin{array}{l}\text { see comments } \\
\text { in their } \\
\text { respective } \\
\text { sections }\end{array}$} \\
\hline Q2 & AUTHOR: Please check that authors' affiliations are correct. & \\
\hline Q3 & AUTHOR: Missing panel A of figure 2 . Please check if figure 2 was captured correctly. & \\
\hline
\end{tabular}




\section{Force spectroscopy reveals the presence of structurally} modified dimers in transthyretin amyloid annular oligomers

Ricardo H. Pires | Maria J. Saraiva | Ana M. Damas | Miklós S.Z. Kellermayer

Transient oligomeric amyloid assemblies remain a challenge for molecular structural studies. Here we show by AFM force spectroscopy that misfolded dimers constitute a key arrangement for the assembly of transthyretin amyloid annular oligomers.

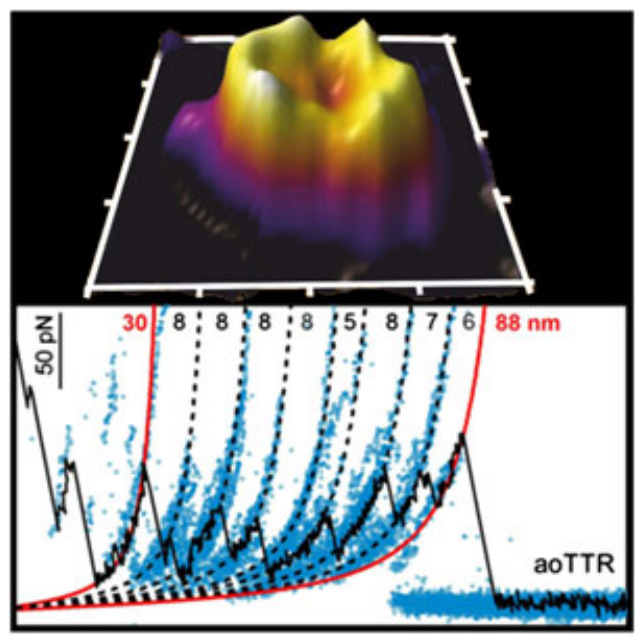


Required software to e-Annotate PDFs: Adobe Acrobat Professional or Adobe Reader (version 7.0 or above). (Note that this document uses screenshots from Adobe Reader $\mathrm{X}$ )

The latest version of Acrobat Reader can be downloaded for free at: http://get.adobe.com/uk/reader/

Once you have Acrobat Reader open on your computer, click on the Comment tab at the right of the toolbar:

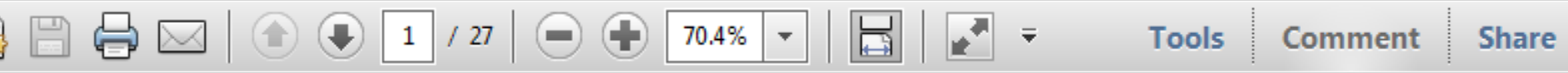

This will open up a panel down the right side of the document. The majority of tools you will use for annotating your proof will be in the Annotations section, pictured opposite. We've picked out some of these tools below:

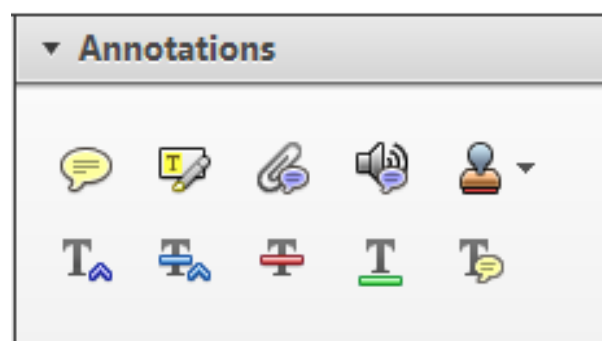

1. Replace (Ins) Tool - for replacing text.

Strikes a line through text and opens up a text box where replacement text can be entered.

How to use it

- Highlight a word or sentence.

- Click on the Replace (Ins) icon in the Annotations section.

- Type the replacement text into the blue box that appears.

Idard tramework for the analysis of $\mathrm{m}$ icy-Nevertheless, it also led to exog،

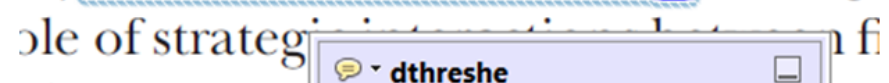
aber of comp 08/06/2011 15:58:17 is that the s1 nain compo: be level, are exc nc

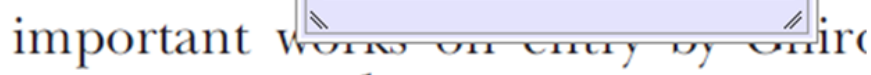
M heneferth) ${ }^{1}$ we anen the "hlarl $\mathrm{l}$

3. Add note to text Tool - for highlighting a section to be changed to bold or italic.

T Highlights text in yellow and opens up a text box where comments can be entered.

\section{How to use it}

- Highlight the relevant section of text.

- Click on the Add note to text icon in the Annotations section.

- Type instruction on what should be changed regarding the text into the yellow box that appears.

namic responses of mark ups ent with the VAR evidence

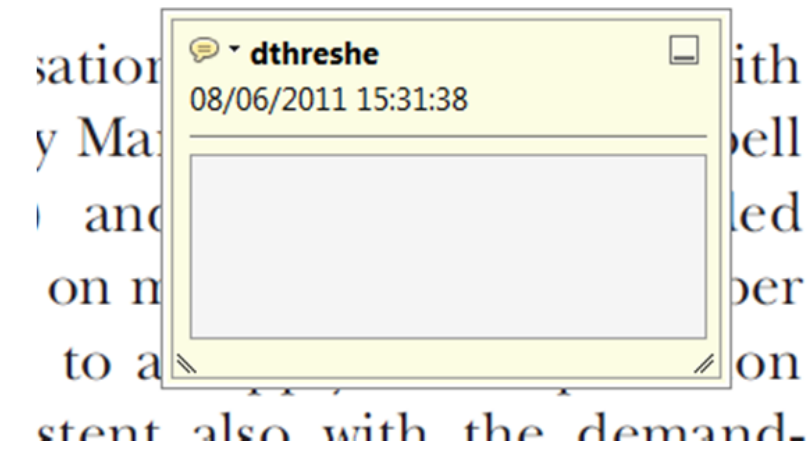

2. Strikethrough (Del) Tool - for deleting text.

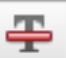

Strikes a red line through text that is to be deleted.

How to use it

- Highlight a word or sentence.

- Click on the Strikethrough (Del) icon in the Annotations section.

there is no room tor extra prohts al c ups are zero and the number of ret) values are not determined by Blanchard and Kiyotaki (1987), sfect competition in general equilil ts of aggregate demand and supply lassical framework assuming monol eph on evorenous number of firme

4. Add sticky note Tool - for making notes at specific points in the text.

Marks a point in the proof where a comment needs to be highlighted.

How to use it

- Click on the Add sticky note icon in the Annotations section.

- Click at the point in the proof where the comment should be inserted.

- Type the comment into the yellow box that appears.

iaisu airu suppiy sirucks. hivsl ui

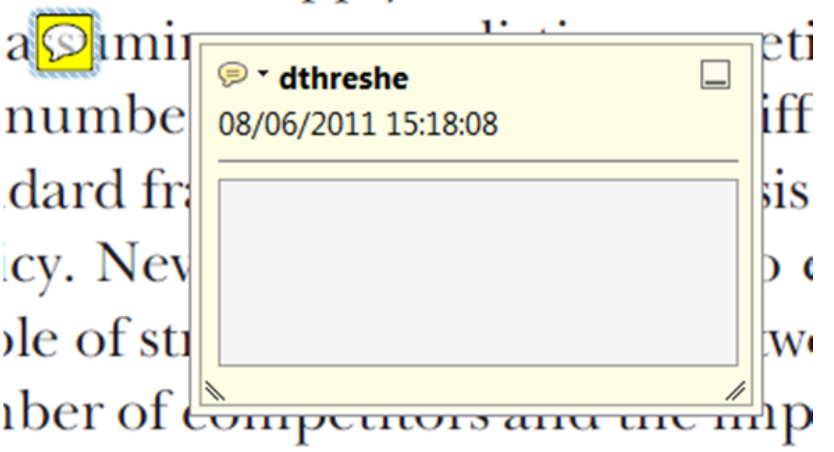

is that the structure of the secto. 
5. Attach File Tool - for inserting large amounts of text or replacement figures.

Inserts an icon linking to the attached file in the appropriate pace in the text.

How to use it

- Click on the Attach File icon in the Annotations section.

- Click on the proof to where you'd like the attached file to be linked.

- Select the file to be attached from your computer or network.

- Select the colour and type of icon that will appear in the proof. Click OK.

E N D

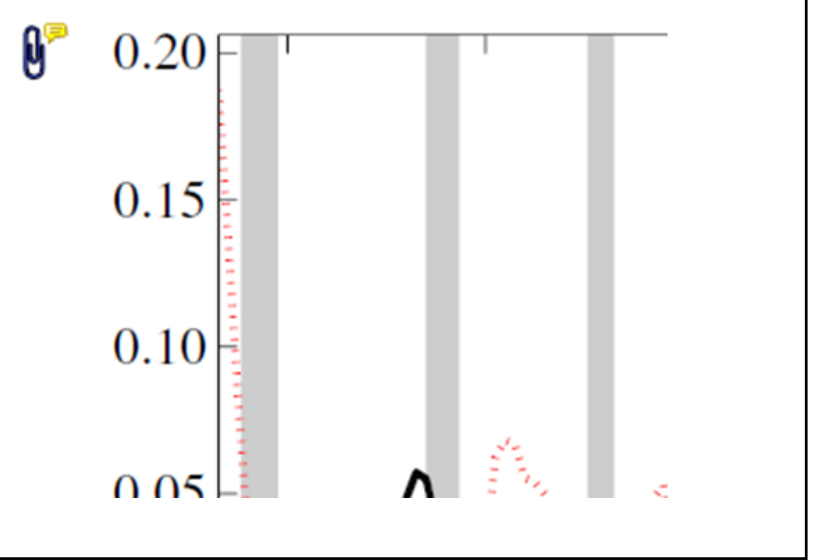

6. Add stamp Tool - for approving a proof if no corrections are required.

- Inserts a selected stamp onto an appropriate place in the proof.

\section{How to use it}

- Click on the Add stamp icon in the Annotations section.

- $\quad$ Select the stamp you want to use. (The Approved stamp is usually available directly in the menu that appears).

- Click on the proof where you'd like the stamp to appear. (Where a proof is to be approved as it is, this would normally be on the first page).

or the business cycie, starting with the on perfect competition, constant ret

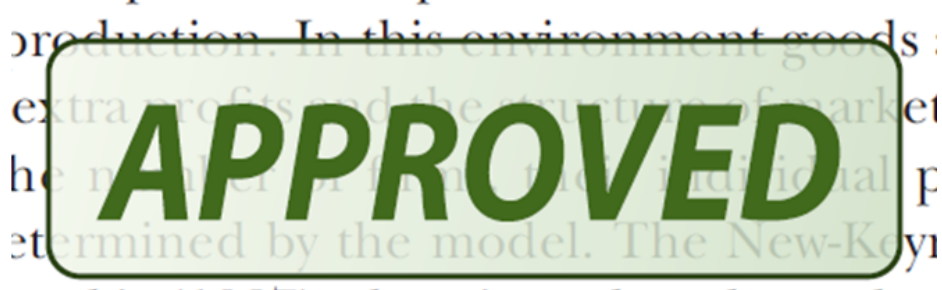
otaki (1987), has introduced produc general equilibrium models with nomin:

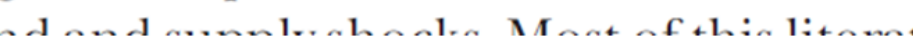

- Drawing Markups

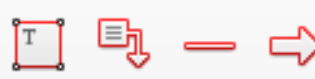

$0 \bigcirc \sqrt{6} \otimes$

\section{How to use it}

- Click on one of the shapes in the Drawing Markups section.

- Click on the proof at the relevant point and draw the selected shape with the cursor.

- To add a comment to the drawn shape, move the cursor over the shape until an arrowhead appears.

- Double click on the shape and type any text in the red box that appears.
7. Drawing Markups Tools - for drawing shapes, lines and freeform annotations on proofs and commenting on these marks.

Allows shapes, lines and freeform annotations to be drawn on proofs and for comment to be made on these marks.

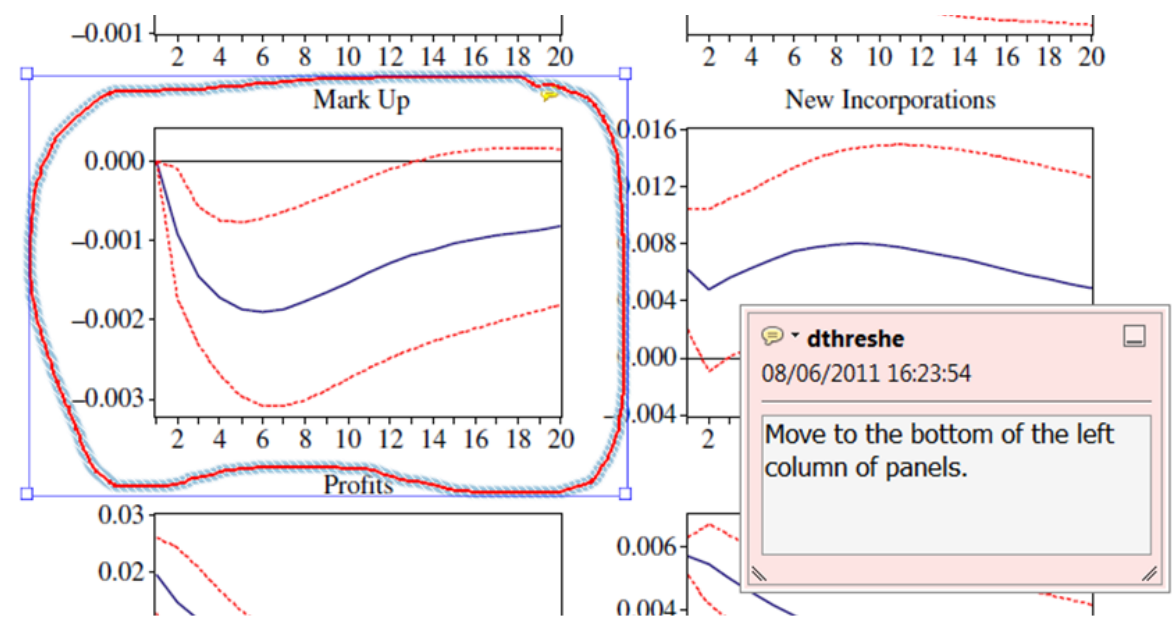

For further information on how to annotate proofs, click on the Help menu to reveal a list of further options:

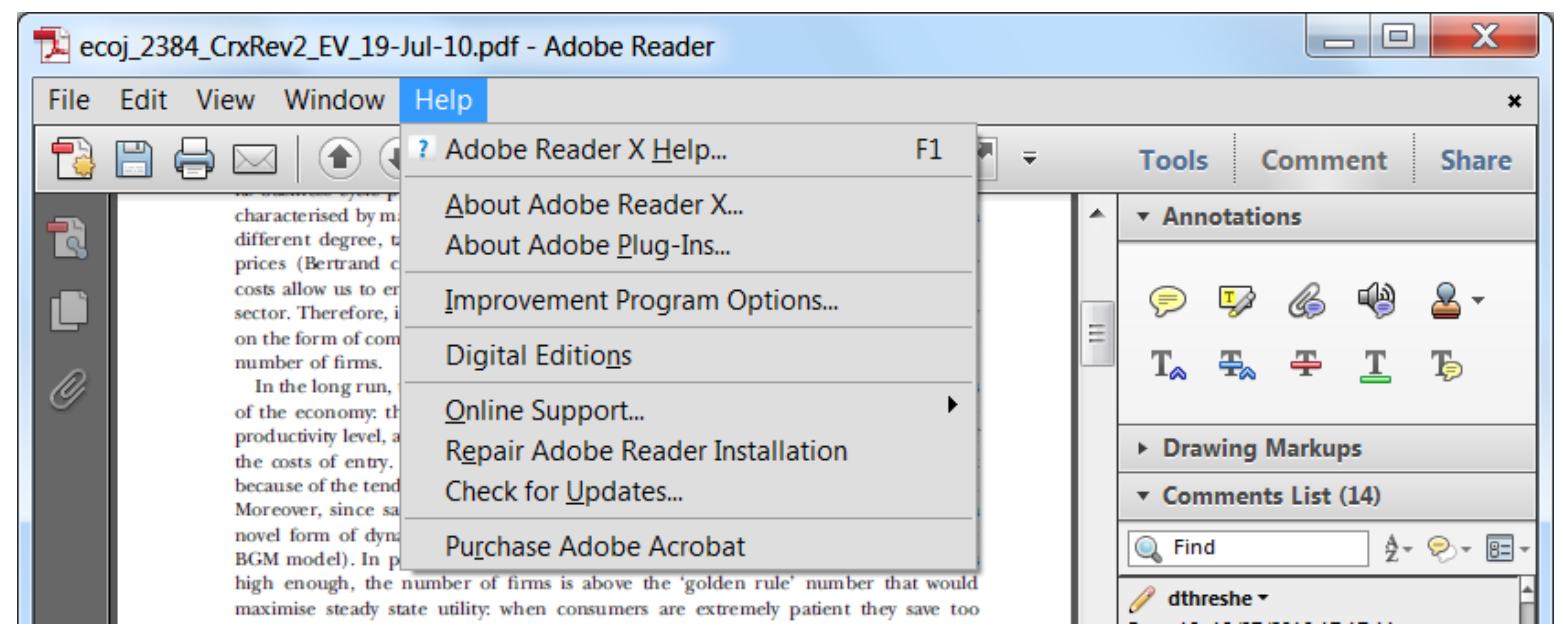

